\title{
Análisis de la evidencia científica de los estudios publicados sobre terapia combinada en Hiperplasia Prostática Benigna
}

\author{
F. Millán Rodríguez \\ Servicio de Urología. Fundación Puigvert. Barcelona. Máster en Metodología de la Investigación en \\ Ciencias de la Salud. Universitat Autónoma de Barcelona.
}

Actas Urol Esp 2005; 29 (8): 725-734

\begin{abstract}
RESUMEN
ANÁLISIS DE LA EVIDENCIA CIENTÍFICA DE LOS ESTUDIOS PUBLICADOS SOBRE TERAPIA COMBINADA EN HIPERPLASIA PROSTÁTICA BENIGNA

Objetivo: El análisis de la evidencia científica de los estudios publicados sobre terapia combinada en Hiperplasia Prostática Benigna (HBP).

Material y métodos: Se han analizado y comparado desde el punto de vista de la Medicina Basada en la Evidencia (MBE) los 5 ensayos clínicos publicados sobre terapia combinada en HBP. Se han analizado las hipótesis y las variables de cada estudio, la validez interna, la relevancia de los resultados, y la validez externa.

Resultados: Cuatro estudios evaluaron los cambios en la sintomatología y en el flujo máximo (Qmax) y sólo uno la progresión de la enfermedad. Los criterios de inclusión y exclusión fueron similares en los 5 estudios. La corta duración, el porcentaje de abandonos, la ausencia de grupo placebo y el sesgo en la dosificación en un brazo de tratamiento fueron las principales causas de escasa o leve validez interna en 3 estudios. La validez externa está afectada en los 5 estudios por la rigidez de los criterios de exclusión y en 3 de ellos por alcanzar el efecto terapéutico con dosis altas del alfabloqueante. Sólo el estudio que tiene un mayor nivel de evidencia científica (MTOPS) proporciona los intervalos de confianza y el número de pacientes a tratar.

Conclusiones: En todos los estudios el incremento del Qmax y la mejora de la sintomatología presentan respectivamente una nula y una moderada relevancia clínica. El estudio MTOPS demuestra que la progresión de la HBP tiene una incidencia baja pero se puede reducir de forma importante con el tratamiento combinado.
\end{abstract}

Palabras clave: Hiperplasia Prostática Benigna. Tratamiento combinado. Medicina Basada en la Evidencia.

\section{ABSTRACT}

\section{ANÁLISIS OF THE SCIENTIFIC EVIDENCE OF THE COMBINATION THERAPY IN BENIGN PROSTATIC} HYPERPLASIA

Objective: The analysis of the scientific evidence of the combination therapy in Benign Prostatic Hyperplasia (BPH).

Methods: 5 published studies about combination therapy in BPH were analysed following the criteria of the Evidence Based Medicine (EBM). Hypothesis, variables, internal validity, results relevance, and the external validity of every study were analysed.

Results: Symptoms changes and maximal flow rate (Qmax) improvement were evaluated in four studies and only one analysed the BPH progression. Inclusion and exclusion criteria were similar in the 5 studies. The main reasons of a poor internal validity in 3 studies were the short follow-up, the missing percentage, the absence of a placebo group and a dosage bias. External validity were decreased in the 5 studies by the exclusion criteria and in 3 of them because high doses of alpha-blocker were given to achieve a therapeutic effect. The study with the highest scientific evidence (MTOPS) is the only that offers confidence intervals and number needed to treat.

Conclusions: The $\operatorname{Bmax}$ and the symptoms improvement found in all the studies has a moderate clinical relevance. MTOPS study shows that BPH progression has a low incidence that can be highly reduced by means of combination therapy.

Keywords: Benign Prostatic Hyperplasia. Combination therapy. Evidence Based Medicine. 
$\mathrm{E}$ $n$ los últimos años se ha generalizado el uso de alfabloqueantes $(\mathrm{AB})^{1-2}$ e inhibidores de la 5-alfa-reductasa (5ARI) ${ }^{3-4}$ en el tratamiento de la hiperplasia prostática benigna (HBP). Cualquier tratamiento de la HBP ha de incidir sobre dos ejes básicos: la mejoría de los síntomas miccionales y la disminución de las complicaciones. Es sobradamente conocido que las dos familias de fármacos tienen un mecanismo de acción diferente. Los $\mathrm{AB}$ actúan disminuyendo el tono adrenérgico y por tanto la obstrucción, y la diferencia entre ellos radica en la uroselectividad de su acción. Por su parte, los 5ARI actúan sobre la regulación del crecimiento y proliferación tisular inhibiendo el crecimiento glandular y por tanto la compresión. Consecuentemente, tiene interés valorar si la acción combinada de ambos tipos de fármacos obtiene mayores beneficios que los tratamientos con monoterapia. A pesar de que son muchos los estudios publicados sobre tratamiento de la HBP mediante $\mathrm{AB}$ o 5ARI, todos los resultados convergen en la misma dirección: la eficacia de la monoterapia. Sin embargo, se han realizado muy pocos estudios sobre terapia combinada en HBP y además, ofrecen resultados contradictorios. Los ensayos clínicos publicados por orden cronológico son los siguientes:

- Estudio VETERANS ${ }^{5}$ : Se analizó la eficacia sobre la sintomatología y el flujo máximo (马max) en 1.229 pacientes divididos en 4 brazos: terazosina, finasteride, placebo y terapia combinada. Concluyeron que la terazosina es efectiva, el finasteride no lo es y la combinación no aporta ninguna ventaja a la monoterapia con terazosina.

- Estudio ALFIN ${ }^{6}$ : Analizan los mismos parámetros en 1.051 pacientes divididos en 3 grupos: alfuzosina, finasteride y terapia combinada. Se concluye que en el $\operatorname{mmax}$ no hay diferencias, mientras que en la mejora de la sintomatología la alfuzosina es efectiva, el finasteride no lo es y la combinación no aporta ninguna mejora añadida.

- Estudio PREDICT ${ }^{7}$ : Se estudian las mismas variables en 1.095 pacientes divididos en 4 grupos: doxazosina, finasteride, placebo y combinación. La conclusión a la que se llega es que la doxazosina es efectiva, el finasteride no lo es y la combinación no aporta nada.

- Estudio SMART ${ }^{8}$ : Estudia 327 pacientes tratados con combinación de tamsulosina y dutas- teride. Tras un tiempo de tratamiento se dividen en 2 grupos: uno sigue el tratamiento combinado y otro sólo dutasteride y placebo. Concluyen que al retirar la tamsulosina empeoran los síntomas urinarios.

- Estudio MTOPS ${ }^{9}$ : Se analiza la progresión de la HBP en 3047 pacientes divididos en 4 grupos: doxazosina, finasteride, placebo y combinación. Concluye que no sólo ambos fármacos disminuyen de forma separada la progresión, sino que además la combinación de ambos disminuye más intensamente la progresión en HBP.

Dado de que se trata de estudios en los que se han usado fármacos diferentes (4 $\mathrm{AB}$ y 2 5ARI) no es fácil realizar un metaanálisis. Sin embargo, sí que tiene interés realizar un análisis crítico pormenorizado de los mismos, comparándolos entre ellos, para conocer si todos tienen el mismo nivel de evidencia científica y para valorar la utilidad real de los fármacos analizados. Este análisis, que es el objetivo de esta revisión, permitirá poner luz sobre los diferentes resultados contradictorios que ofrecen estos estudios. La metodología de análisis empleada es la descrita previamente por la Medicina Basada en la Evidencia ${ }^{10}$, y también por los criterios de análisis de un ensayo clínico en HBP publicados previamente en esta revista ${ }^{11}$.

\section{ANÁLISIS DE LA HIPÓTESIS Y LAS VARIABLES}

El primer aspecto a estudiar en cualquier ensayo clínico es el análisis de la hipótesis del mismo. En primer lugar, hay que valorar si el estudio aporta algo nuevo a lo que ya se conoce a través del estudio de la literatura o de la experiencia propia. Esto nos proporcionará una idea de la originalidad o del posible impacto que pueda tener dicho estudio, ya que no es lo mismo plantear una hipótesis nueva que repetir una idea ya evaluada previamente con diferentes tratamientos o pacientes. Al igual que en la mayoría de estudios publicados previamente con monoterapia en HBP, en 3 de los trabajos publicados sobre terapia combinada en $\mathrm{HBP}^{5-7}$ lo que se analiza es la eficacia de los distintos tratamientos sobre el flujo máximo y la sintomatología miccional. Sin embargo, los otros dos estudios analizan hipótesis más originales ya que el estudio SMART 
valora el deterioro de la sintomatología tras la retirada del $\mathrm{AB}^{8}$ y el estudio MTOPS el posible retraso o prevención de la progresión clínica de la $\mathrm{HBP}^{9}$.

Una vez presentada la hipótesis o el objetivo del estudio, ha de evaluarse si éste ha sido correctamente definido. Para ello ha de analizarse cuáles son las variables que se han usado y de qué forma se han medido. En los 3 trabajos que evalúan la eficacia ${ }^{5-7}$ las principales variables analizadas fueron los cambios en el flujo máximo y en la sintomatología mediante el cuestionario $\mathrm{AUA}^{5}$ o I-PSS ${ }^{6-7}$. Estas dos variables son las que se han usado clásicamente para valorar la eficacia de cualquier tratamiento en HBP y en principio parecen adecuadas para los objetivos de estos estudios. En el estudio SMART ${ }^{8}$ la variable principal fue la respuesta a la pregunta “ ¿En las 2 últimas semanas, se ha sentido usted mejor, peor o igual con respecto a sus síntomas urinarios, que en la última visita?" y la variable secundaria los cambios en el cuestionario I-PSS. Por ello, cabe cuestionarse que si el objetivo es evaluar el deterioro de la sintomatología tras retirar el $\mathrm{AB}$, por qué como variable principal, en vez de usar la respuesta a la pregunta antes mencionada, no se usa el cuestionario I-PSS que al fin y al cabo, es uno de los principales cuestionarios de sintomatología validados internacionalmente. Por otro lado, en el estudio MTOPS ${ }^{9}$ el análisis de los cambios en el flujo máximo y el cuestionario AUA no son las variables principales ya que lo que se analiza es la progresión en HBP. Este es un concepto novedoso que los autores definieron como la presencia de cualquiera de estos acontecimientos: incremento igual o superior a 4 puntos en el cuestionario AUA, retención urinaria aguda, insuficiencia renal, infección urinaria de repetición e incontinencia urinaria. En este caso, los autores han definido unos criterios claros de lo que entienden por progresión, basados en el empeoramiento de la sintomatología o en la aparición de complicaciones.

Se puede concluir que 3 estudios $^{5-7}$ plantean objetivos clásicos basados en variables ampliamente aceptadas, mientras que los otros dos nacen de unos objetivos originales y por ello más novedosos pero basados en unas variables definidas por primera vez en dichos estudios ${ }^{8-9}$.

\section{ANÁLISIS DE LA VALIDEZ INTERNA}

En un ensayo clínico los diferentes resultados hallados en cada brazo de tratamiento pueden ser debidos a la acción propia de los diferentes tratamientos recibidos o bien, a una mala jugada del azar o a la presencia de sesgos. Es precisamente esto lo que evalúa la validez interna. De este modo, un estudio con una buena validez interna nos garantizará que los resultados del mismo se deben a la diferente eficacia de los tratamientos administrados y no al azar o a cualquier otro elemento distorsionador. La validez interna depende principalmente de estos 5 elementos:

- asignación aleatoria de los sujetos.

- seguimiento de los pacientes.

- estrategia del análisis por intención de tratar.

- enmascaramiento.

- comparabilidad inicial de los grupos.

Los 5 estudios cumplen la asignación aleatoria de los sujetos y el enmascaramiento (doble ciego). El estudio SMART ${ }^{8}$ no informa al respecto, pero en los otros 4 el análisis se ha realizado por intención de tratar. Otro aspecto importante es el análisis de la comparabilidad inicial de los distintos grupos, ya que aunque la asignación sea aleatoria puede pasar que hayan desequilibrios en algún grupo respecto a alguna variable que pueda influir en el resultado final, como por ejemplo el volumen prostático. Con respecto a esta cuestión, todos los grupos son comparables al inicio en los 5 estudios.

En lo referente al seguimiento de los pacientes, es de prever un mayor número de abandonos cuanto más largo es el estudio. Como la duración de los estudios abarca desde los 6 meses hasta los 4,5 años, una forma de compararlos es analizando el número de abandonos producidos cada 6 meses. Tal como muestra la Tabla 1 se observa que dicho porcentaje oscila entre el $2,5 \%$ del estudio MTOPS ${ }^{9}$ hasta el 13 o 14,5\% de los estudios ALFIN ${ }^{6}$ y PREDICT $^{7}$ respectivamente. Sin embargo, no sólo tiene interés conocer el porcentaje de abandonos sino la causa de los mismos, ya que no tiene el mismo significado abandonar por desinterés o cambio de domicilio del paciente que porque el paciente note falta de eficacia o algún efecto adverso. Esta información esta disponible en los estudios Veterans ${ }^{5}$ y PREDICT $^{7}$ y parcialmente en el estudio MTOPS $^{9}$. 
Tabla 1

Características principales de los estudios

\begin{tabular}{|c|c|c|c|c|c|c|c|c|}
\hline & № casos & $\begin{array}{c}\text { Duración } \\
\text { media }\end{array}$ & $\begin{array}{c}\text { Porcentaje } \\
\text { total } \\
\text { abandonos }\end{array}$ & $\begin{array}{c}\text { Porcentaje } \\
\text { abandonos } \\
\text { cada } 6 \text { meses }\end{array}$ & $\begin{array}{l}\text { Objetivo } \\
\text { primario }\end{array}$ & $\begin{array}{l}\text { Validez } \\
\text { interna }\end{array}$ & Relevancia & Validez \\
\hline VETERANS & 1129 & 1 año & $18 \%$ & $9 \%$ & $\begin{array}{l}\text { Qmax } \\
\text { AUA }\end{array}$ & ++ & $\begin{array}{c}\text { Qmax } 1,8 \mathrm{ml} / \mathrm{s} \\
\text { AUA 3,6 puntos } \\
10 \mathrm{mg}\end{array}$ & $\begin{array}{c}\text { No NNT } \\
80 \% \text { terazosina }\end{array}$ \\
\hline PREDICT & 1095 & 1 año & $29 \%$ & $14,5 \%$ & $\begin{array}{l}\text { Qmax } \\
\text { I-PSS }\end{array}$ & $\begin{array}{c}+ \\
\text { Dosificación } \\
\text { Doxazosina } \\
\text { Abandonos }\end{array}$ & $\begin{array}{l}\text { Qmax } 2,4 \mathrm{ml} / \mathrm{s} \\
\text { I-PSS } 2,8 \text { puntos }\end{array}$ & $\begin{array}{c}\text { No NNT } \\
60 \% \text { doxazosina } \\
8 \mathrm{mg}\end{array}$ \\
\hline SMART & 327 & 9 meses & - & $9 \%$ & Pregunta & $\begin{array}{c}+ \\
\text { Pregunta }\end{array}$ & - & - \\
\hline MTOPS & 3047 & 4,5 años & $22,5 \%$ & $2,5 \%$ & Progresión & +++ & $\begin{array}{l}\text { Gran reducción } \\
\text { Riesgo } \\
\text { Incidencia baja } \\
\text { progresión }\end{array}$ & $\begin{array}{c}\text { NNT } \\
70 \% \text { doxazosina } \\
8 \mathrm{mg}\end{array}$ \\
\hline
\end{tabular}

Otro aspecto importante es el análisis del tamaño muestral, que osciló entre $327^{8}$ y $3.047^{9}$. Habitualmente, se piensa que a mayor tamaño muestral mejor es el estudio y esto no es exactamente así. Un ensayo clínico ha de tener una muestra lo suficientemente grande para asegurar una buena potencia del estudio y lo suficientemente pequeña para que sea lo más sencillo y barato de realizar. Este número no es arbitrario, sino que ha de calcularse en función de la potencia y de las pruebas estadísticas que se usarán en el estudio y también de la diferencia mínima a detectar entre los distintos grupos del estudio. Así, un estudio con menos casos de los necesarios puede que no tenga la suficiente potencia para detectar diferencias entre los distintos tratamientos en caso de que existan. Por el contrario, si la muestra es superior a la necesaria lo único que se conseguirá es que se necesitarán más centros, con lo que se alargará y encarecerá el estudio sin reportar ninguna ventaja adicional. En ninguno de estos ensayos clínicos se justifica en el apartado de "Material y Métodos" el por qué se ha escogido sus respectivos tamaños muestrales.
Existen otros elementos que disminuyen la validez interna de algunos de los estudios. En primer lugar, se considera que un ensayo clínico de una patología como la HBP, que presenta una progresión lenta con altibajos en su sintomatología, ha de tener una duración mínima de un año y en dos de los estudios ésta es sólo de 6 meses $^{6,8}$. Además, en uno de ellos, el estudio ALFIN ${ }^{6}$, no existe grupo control con placebo.

Finalmente, existe otro hecho que cuestiona la validez interna del estudio PREDICT ${ }^{7}$. Dado que el efecto adverso más importante de la doxazosina es la aparición de mareo o hipotensión, es lógico que tal como se realizó en el estudio MTOPS $^{9}$ tanto la dosis de doxazosina o del placebo de doxazosina se vaya aumentando o disminuyendo a $4 \mathrm{u} 8 \mathrm{mg} /$ día de forma ciega en función de la aparición de mareo o no. No obstante, en el estudio PREDICT ${ }^{7}$ el apartado "Tratamiento" dentro de la sección de "Material y Métodos" informa de que a la semana 10, los pacientes aumentarán su dosis de doxazosina de 4 a $8 \mathrm{mg}$ si su IPSS no ha disminuido un $30 \%$ o su Qmax no ha aumentado $3 \mathrm{ml} / \mathrm{s}$. Esto es totalmente incorrecto, porque la modificación de la dosis no se realiza para evitar abandonos por el 
efecto adverso, sino por la consecución o no del objetivo primario del estudio, que es la eficacia del tratamiento evaluada mediante los cambios en el IPSS o en el Gmax.

\section{RELEVANCIA DE LOS RESULTADOS}

Se ha comentado cómo un estudio con una buena validez interna nos garantiza con una alta probabilidad que los resultados obtenidos se pueden atribuir al efecto de los diferentes tratamientos recibidos. En este apartado, van a analizarse los resultados de los distintos estudios, pero ha de tenerse siempre presente que en aquellos estudios con problemas de validez interna, se ha de ser más cauteloso para aceptar los resultados ya que pueden no ser consecuencia directa del tratamiento recibido. Para analizar la relevancia de los resultados han de valorarse los 4 primeros escalones de la pirámide de la Figura 1. En esta pirámide, que analiza los niveles de información, se observa que a medida que subimos un escalón disponemos de mayor información clínica.

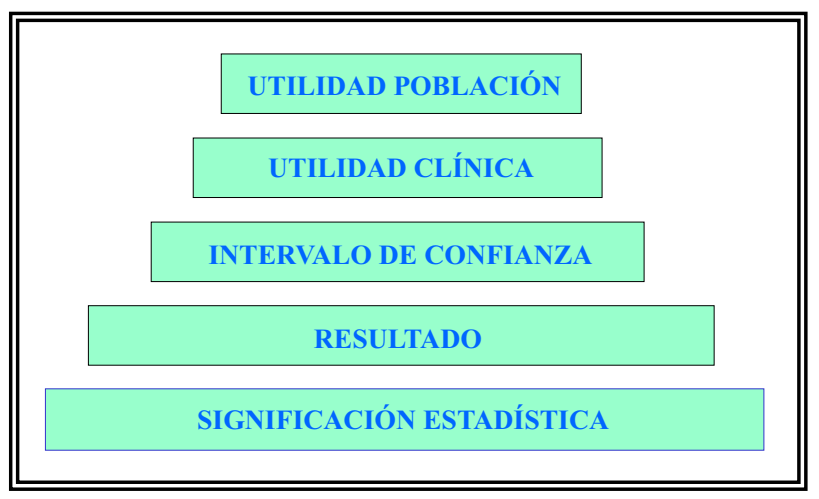

FIGURA 1. Niveles de información

\section{Significación estadística}

Aunque no aparezcan detalladas en ninguno de los ensayos clínicos analizados, en todo estudio ha de haber una hipótesis nula y una alternativa. La hipótesis nula es la que se pretende rechazar, en nuestro caso vendría a ser: "El fármaco $\mathrm{X}$ tiene la misma eficacia que el placebo en el tratamiento de la HBP". Mientras que la hipótesis alternativa es la que se pretende demostrar y sería: "El fármaco $\mathrm{X}$ tiene distinta eficacia que el placebo en el tratamiento de la HBP”. Por otro lado, puede ocurrir que siendo cierta la hipótesis nula (o sea que el fármaco X no sea más eficaz que el placebo) el estudio acabe aceptando la hipótesis alternativa (que el fármaco X es más eficaz que el placebo). Este constituye el llamado error alfa $(\alpha)$. En Ciencias de la Salud se acepta como máximo un error $\alpha$ del $5 \%$, por ello el corte de significación estadística se ha establecido en $\mathrm{p}=0,05$. En definitiva, lo que quiere decir que una p es estadísticamente significativa, es que la probabilidad de que por azar se concluya erróneamente que el fármaco X es más eficaz que el placebo sin que realmente sea así, es inferior al 5\%. $\mathrm{El}$ valor $\mathrm{p}$ es un valor que sólo tiene interpretación estadística pero que no aporta información sobre la relevancia clínica del resultado. Un error habitual es considerar que el fármaco de un estudio con una $\mathrm{p}$ muy pequeña (por ejemplo $\mathrm{p}=0,00001$ ) es mucho más eficaz que el fármaco de un estudio con una $\mathrm{p}$ superior (por ejemplo $\mathrm{p}=0,049$ ).

Del análisis de los 5 artículos se desprende que en todos ellos las pruebas estadísticas fueron estadísticamente significativas, excepto en la comparación de los cambios en el Gmax entre alfuzosina y finasteride en el estudio ALFIN ya que fueron estadísticamente no significativos ${ }^{6}$.

\section{Resultado e intervalo de confianza}

Una vez que se ha aceptado la significación estadística de las hipótesis estudiadas ha de analizarse el resultado obtenido. Supongamos que el resultado es que el fármaco $\mathrm{X}$ aumenta el $\mathrm{Qmax}$ una media de $3 \mathrm{ml} / \mathrm{s}$ respecto al placebo. ¿Quiere esto decir que cada paciente que tome el fármaco $\mathrm{X}$ aumentará su Qmax exactamente $3 \mathrm{ml} / \mathrm{s}$ ? ¿Si se volviera a repetir el estudio con una muestra de las mismas características el resultado volvería a ser exactamente $3 \mathrm{ml} / \mathrm{s}$ ? Obviamente, la respuesta a estas dos preguntas es negativa, pero si diéramos el fármaco $\mathrm{X}$ a 100 pacientes o repitiéramos el mismo estudio 100 veces se observaría que en 95 casos el aumento de 3 max oscilaría entre dos valores alrededor de $3 \mathrm{ml} / \mathrm{s}$ (por ejemplo entre 1 y $5 \mathrm{ml} / \mathrm{s}$ ). Esta es la información que aporta el intervalo de confianza del $95 \%\left(\mathrm{IC}_{95 \%}\right)$ y tal como se observa en la Figura 1 se encuentra un escalón por encima del resultado ya que aporta más información clínica. Siguiendo el mismo ejemplo, el valor del resultado sólo indica que con el fármaco X el Qmax aumentará una media de $3 \mathrm{ml} / \mathrm{s}$ 
pero el $\mathrm{IC}_{95}$ aporta más información clínica, ya que nos dice que el $95 \%$ de los pacientes tratados con el fármaco X su Qmax aumentará de 1 a 5 $\mathrm{ml} / \mathrm{s}$. Por ello un estudio correctamente realizado ha de aportar no sólo el resultado obtenido sino también su $\mathrm{IC}_{95 \%}$. De los 5 estudios realizados el único que aporta los $\mathrm{IC}_{95 \%}$ de la variable principal es el estudio MTOPS ${ }^{9}$ y el SMART $^{8}$ sólo en una de ellas.

\section{Utilidad clinica}

Se apuntaba anteriormente que la relevancia clínica o la utilidad de un fármaco no viene dada por la significación estadística del estudio. La utilidad clínica de un fármaco o una intervención se evalúa analizando la repercusión clínica que tiene el resultado y el intervalo de confianza obtenidos. La Tabla 2 ofrece una comparación de los resultados de cada estudio y en el caso de la variable Gmax que es el objetivo primario de los estudios VETERANS ${ }^{5}$, ALFIN ${ }^{6}$ y PREDICT ${ }^{7}$ y objetivo secundario del estudio MTOPS $^{9}$, se observa que la diferencia $\mathrm{Qmax}$ entre cada fármaco individual o combinado respecto a placebo oscila de $0,9 \mathrm{ml} / \mathrm{s}$ a $2,4 \mathrm{ml} / \mathrm{s}$. Todos estos resultados son estadísticamente significativos pero cabe cuestionarse si realmente el paciente va a notar una mejoría de su micción por el mero hecho de aumentar 2,4 ml/s su Qmax. Por otro lado, hay trabajos previos que demuestran que la variabilidad intraindividual del $3 \max$ cuando se realiza una flujometría es de $2 \mathrm{ml} / \mathrm{s}^{12}$. ¿Puede considerarse un aumento de $\mathrm{Qmax}$ de 0,9 o $2,4 \mathrm{ml} / \mathrm{s}$ clínicamente relevante cuando la variabilidad intraindividual es de $2 \mathrm{ml} / \mathrm{s}$ ? El caso de la mejora media del $\mathrm{Qmax}$ en el tratamiento de la HBP es un ejemplo de cómo una variable estadísticamente significativa no se acompaña de una relevancia clínica importante.

La siguiente variable estudiada es la mejoría de la sintomatología medida mediante el cuestionario IPSS o el de la AUA. En los 4 estudios en que se valora, se observa también una diferencia estadísticamente significativa de los distintos tratamientos con respecto al placebo, pero el resultado es que la mejora máxima observada es de 3,6 puntos. Para valorar la utilidad clínica de este hecho tenemos que responder a esta pregunta: ¿Sobre una puntuación total de 35 una mejora de puntuación de 1 a 3,6 tiene gran relevancia clínica? Es otro ejemplo de cómo la relevancia de un tratamiento no viene establecida por la significación estadística sino por la respuesta a preguntas que nos hemos de plantear como clínicos.

En cuanto a la variable "Progresión" que es la variable principal del estudio MTOPS $^{9}$ se obtiene una reducción del riesgo relativo del 34\% al 39\% en monoterapia y que casi se dobla (66\%) cuando se administra tratamiento combinado. Para valorar la utilidad del tratamiento combinado en la prevención de la progresión en HBP ha de res-

\section{Tabla 2}

Resultados con sus intervalos de confianza de las variables principales

\begin{tabular}{|c|c|c|c|c|c|}
\hline Estudio & Fármacos comparados & Qmax & IPSS / AUA & Empeoramiento clínico & $\begin{array}{l}\text { Disminución del Riesgo } \\
\text { Progresión Clínica Global }\end{array}$ \\
\hline \multirow[t]{2}{*}{ VETERANS } & Terazosina vs Placebo & $1,3 \mathrm{ml} / \mathrm{s}$ & 3,5 puntos & - & - \\
\hline & $\begin{array}{c}\text { Terazosina + Finasteride } \\
\text { vs Placebo }\end{array}$ & $1,8 \mathrm{ml} / \mathrm{s}$ & 3,6 puntos & - & - \\
\hline ALFIN & Alfuzosina vs Finasteride & No significativo & 1,1 puntos & - & - \\
\hline \multirow[t]{2}{*}{ PREDICT } & $\begin{array}{l}\text { Doxazosina vs Placebo } \\
\text { Doxazosina + Finasteride }\end{array}$ & $2,2 \mathrm{ml} / \mathrm{s}$ & 2,6 puntos & - & - \\
\hline & vs Placebo & $2,4 \mathrm{ml} / \mathrm{s}$ & 2,8 puntos & - & - \\
\hline SMART & Dutasteride + Tamsulosina & - & - & $14 \%$ & - \\
\hline \multirow[t]{3}{*}{ MTOPS } & Doxazosina & $1,2 \mathrm{ml} / \mathrm{s}$ & 2 puntos & - & $39 \%\left(\mathrm{IC}_{95 \%}=20-53\right)$ \\
\hline & Finasteride & $0,9 \mathrm{ml} / \mathrm{s}$ & 1 punto & - & $34 \%\left(\mathrm{IC}_{95 \%}=14-50\right)$ \\
\hline & Doxazosina + Finasteride & $2,4 \mathrm{ml} / \mathrm{s}$ & 3 puntos & - & $66 \%\left(\mathrm{IC}_{95 \%}=54-76\right)$ \\
\hline
\end{tabular}


ponderse esta pregunta: ¿Una reducción del riesgo relativo de progresión del $66 \%$ es clínicamente importante? Es evidente que estamos ante una importante reducción del riesgo pero también ha de tenerse en cuenta cuál es la incidencia real de progresión. Esta viene dada por la incidencia de progresión hallada en el grupo placebo y que es de un $11,5 \%$. No tiene la misma repercusión clínica reducir un $66 \%$ el riesgo de un evento que ocurre en el $11,5 \%$ de los casos que de un evento que ocurriera, por ejemplo, en el $50 \%$ de los casos.

\section{ANÁLISIS DE LA VALIDEZ EXTERNA}

Un ensayo clínico evalúa la efectividad de un determinado tratamiento a partir de los resultados obtenidos al aplicarlo en una muestra de unas características determinadas. Sin embargo, que un tratamiento haya demostrado su efectividad en la muestra estudiada no garantiza que se pueda generalizar dicha efectividad a la población general. Precisamente, lo que evalúa la validez externa es la aplicabilidad práctica de los resultados obtenidos en la población. El análisis de la validez externa se basa en la evaluación de estos tres elementos:

- diferencias entre los pacientes seleccionados y la población a tratar.

- modificación del pronóstico.

- relación entre beneficios, seguridad y coste.

Solamente se puede extrapolar los resultados de un estudio a una población que tenga las mismas características que la muestra que participó en el estudio. Las características de los pacientes seleccionados en el estudio vienen definidas por los criterios de inclusión y exclusión. Los 5 estudios presentan unos criterios bastante similares; en la Tabla 3 se resumen los principales criterios de inclusión observándose que en cuanto a @max se incluyen pacientes con un $\mathrm{Qmax}$ de 4 ó 5 a 15 $\mathrm{ml} / \mathrm{s}$. En lo referente a la sintomatología, en 3 de ellos se requiere una puntuación mínima de $7 \mathrm{u}$ $8^{5,6,9}$ y en los otros dos de $12^{7-8}$. Estas características son similares a las que puede tener la población de pacientes que trataremos por HBP. Sin embargo, esta buena aplicabilidad de los resultados de los estudios a la población general se ve afectada por los criterios de exclusión ya que en todos los estudios fueron muy restrictivos. De
Tabla 3

Criterios de inclusión

\begin{tabular}{lcc}
\hline Estudio & Sintomatología & Qmax \\
\hline VETERANS & AUA $>8$ & $4-15 \mathrm{ml} / \mathrm{s}$ \\
ALFIN & IPSS $>7$ & $5-15 \mathrm{ml} / \mathrm{s}$ \\
PREDICT & IPSS $>12$ & $5-15 \mathrm{ml} / \mathrm{s}$ \\
SMART & IPSS $>12$ & $4-15 \mathrm{ml} / \mathrm{s}$ \\
MTOPS & AUA $8-30$ & $4-15 \mathrm{ml} / \mathrm{s}$ \\
\hline
\end{tabular}

este modo, en la mayoría de ellos se excluyeron pacientes con insuficiencia renal leve, historia de infecciones urinarias, trastornos de la coagulación, hiperreflexia del detrusor, cardiopatías, accidentes vasculocerebrales, o polimedicados. En el estudio MTOPS los criterios de exclusión únicamente fueron intervención quirúrgica previa para $\mathrm{HBP}$, tensión arterial menor de 90/70 $\mathrm{mm} \mathrm{Hg}$ y PSA $>10 \mathrm{ng} / \mathrm{ml}$. Así como en la práctica clínica diaria es fácil encontrar pacientes afectos de HBP que cumplan los criterios de inclusión, es muy difícil encontrar casos que no presenten ninguno de los criterios de exclusión. Este hecho disminuye la validez externa de los estudios ya que las características de la muestra utilizada en los estudios es bastante distinta a la de la población general de pacientes afectos de HBP. No obstante, esta situación es muy frecuente en la mayoría de ensayos clínicos, urológicos o no, ya que lo que se pretende conseguir con unos criterios de exclusión tan restrictivos es una mayor homogeneidad de la muestra para alcanzar una mayor validez interna.

El siguiente punto a analizar de la validez externa es si el tratamiento consigue una modificación del pronóstico de la enfermedad, o sea su utilidad cuando se aplica a la población general. Nos encontramos ante el último escalón de la pirámide presentada en la Figura 1. La evaluación de la utilidad en la población se realiza con el llamado número de pacientes a tratar o NNT (number needed to treat). El NNT es el número de pacientes que ha de tratarse para evitar la aparición de un evento determinado (enfermedad, complicación, progresión). El estudio MTOPS ${ }^{9}$ es el único que ofrece esta información, ya que concluye que el NNT de la terapia combinada para 
evitar la progresión es 8,4. Esto quiere decir que hay que tratar 8,4 casos de HBP con combinación de doxazosina y finasteride para evitar un caso de progresión. El NNT mejora hasta casi 5 cuando la próstata es mayor de $40 \mathrm{~g}$ o cuando el paciente tiene niveles de PSA superiores a 4. Ante estos resultados cabe plantearse la siguiente cuestión: ¿es bueno el NNT observado en el estudio MTOPS?. No existe ningún valor concreto aceptado como límite a partir del cual pueda considerarse un NNT como bueno o no. Únicamente cabe compararlo con el NNT de tratamientos de otras patologías en que su uso sea ampliamente aceptado. Así existen dos fármacos cuyo uso está ampliamente aceptado y que son el alendronato en la osteoporosis ${ }^{9}$ y la simvastatina en la hipercolesterolemia ${ }^{10}$. El tratamiento con alendronato presenta una NNT de 15 para evitar una fractura en pacientes afectas de osteoporosis ${ }^{13}$ y la simvastatina una NNT de 25 para evitar un evento cardiovascular adverso en pacientes con hipercolesterolemia ${ }^{14}$. Si se comparan estos NNT con el del estudio MTOPS ${ }^{9}$, se observa por un lado, que el NNT de 8,4 en el tratamiento combinado en HBP es mucho mejor que el de las otras dos patologías, pero por otro lado, la patología que previene (progresión en HBP definida como empeoramiento clínico o aparición de complicaciones) tiene menor impacto en morbilidad que las otras dos patologías (fractura ósea o infarto de miocardio).

$\mathrm{El}$ último aspecto a valorar de la validez externa de un tratamiento es la seguridad y el coste. $\mathrm{El}$ efecto adverso más frecuente atribuible a un $\mathrm{AB}$ es la hipotensión postural o el mareo, mientras que el de un 5ARI es la disfunción eréctil. Hay que ser cautelosos a la hora de interpretar la disfunción eréctil como efecto adverso, ya que todos los estudios valoran su aparición durante el ensayo clínico pero no hay información sobre la prevalencia previa al inicio del estudio. Por ello y al no ser un criterio de exclusión (al contrario de la hipotensión o el mareo) no podemos conocer cuántos pacientes que presentan disfunción eréctil la presentaban ya al inicio del estudio. En cualquier caso, el tratamiento combinado no aumenta de forma significativa el porcentaje de efectos adversos que causa la monoterapia por separado. En cuanto al estudio de costes, ninguno de los ensayos clínicos publicados presenta un estudio sobre el impacto económico que comporta seguir una pauta terapéutica determinada.
Finalmente, no hay que olvidar que para conseguir en la población una eficacia similar a la encontrada en la muestra del estudio, ha de administrarse el tratamiento en las mismas condiciones que en el ensayo clínico. Así, hay que tener presente que la eficacia de los distintos tratamientos combinados se obtuvo administrando dosis altas de $A B$, superiores a las que se suelen pautar en la práctica clínica habitual. En el estudio VETERANS ${ }^{5}$ el $80 \%$ de pacientes recibieron dosis de $10 \mathrm{mg}$ de terazosina, y en el estudio PREDICT $^{7}$ y MTOPS $^{9}$ el $60 \%$ y el $70 \%$ de los pacientes recibieron respectivamente dosis de 8 mg de doxazosina.

\section{DISCUSIÓN}

A pesar de que todos los estudios analizados son ensayos clínicos y por lo tanto se encuentran en el nivel más alto de evidencia científi$\mathrm{ca}^{10}$, tras el análisis pormenorizado de los mismos se puede comprender que no todos tienen el mismo nivel de evidencia científica (Figura 2). No existe ningún método que nos permita otorgar una puntuación de evidencia para cada estudio, pero el análisis de la validez interna sí que nos puede orientar al respecto (Tabla 1). De este modo, desde nuestro punto de vista, los estudios con menor evidencia científica son el ALFIN $^{6}$ y el SMART ${ }^{8}$. Ambos tienen sólo una duración de 6 meses y en el caso del estudio ALFIN $^{6}$ no hay grupo placebo y tiene un elevado porcentaje de abandonos. Por otro lado, el objetivo primario del estudio SMART $^{8}$ se basa en una pregunta y no en uno de los cuestionarios

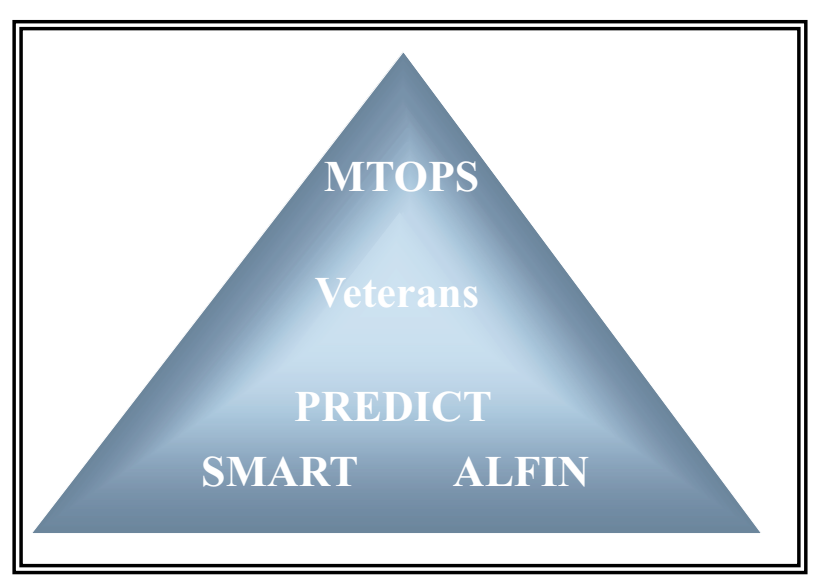

FIGURA 2. Nivel de evidencia cientifica de los estudios de terapia combinada en HBP. 
de sintomatología aceptados internacionalmente. Según nuestro criterio, el estudio PREDICT ${ }^{7}$ tiene algo más de evidencia ya que es de un año de duración aunque no obstante, presenta un gran número de abandonos y además tal como ya se comentó previamente, la dosificación de la doxazosina se realiza en función del efecto terapéutico obtenido. Los dos estudios con mayor evidencia científica son el VETERANS $^{5}$ y el MTOPS $^{9}$, sobresaliendo este último por el diseño, la duración media de 4,5 años, el pequeño porcentaje relativo de abandonos y por ser el único que ofrece el NNT.

Los estudios previos a MTOPS ${ }^{9}$ concluyeron que el añadir finasteride a un $\mathrm{AB}$ no proporciona mayor eficacia que la conseguida con el $\mathrm{AB}$ solo. No obstante, el estudio MTOPS $^{9}$ concluye lo contrario, o sea, que no sólo el $\mathrm{AB}$ o el finasteride son eficaces como monoterapia, sino que la eficacia aumenta considerablemente cuando se pautan de forma combinada. A nuestro entender, esto se debe principalmente a dos hechos. En primer lugar, el estudio MTOPS $^{9}$ plantea un objetivo diferente, ya que mientras en los estudios previos, el objetivo era valorar los cambios en la sintomatología o en el $\mathrm{Bmax}$, en el estudio MTOPS ${ }^{9}$ el objetivo fue evaluar la progresión de la enfermedad. Por otro lado, otro factor es la diferente duración de los estudios. Tal como se muestra en la Figura 3, la eficacia del tratamiento combinado en la prevención de la progresión aumenta a medida que transcurre el tiempo. Los estudios previos tuvieron una duración de 6 meses ó 1 año, tiempo aún insuficiente para constatar esta tendencia a favor del tratamiento combinado que se hace evidente en el estudio MTOPS ${ }^{9}$ al tener una duración media de 4,5 años.

Una vez valorado el nivel de evidencia científica de los diferentes estudios, es preciso analizar la relevancia clínica de los tres estudios con mayor nivel de evidencia (Tabla 1). Tanto el estudio VETERANS ${ }^{5}$ como el PREDICT ${ }^{7}$ llegan a la misma conclusión: que el tratamiento con terazosina o doxazosina respectivamente, consiguen una mejora estadísticamente significativa del $\mathrm{Qmax}$ y de la sintomatología. Sin embargo, la relevancia clínica del aumento medio de $\operatorname{Qmax}\left(1,8 \mathrm{ml} / \mathrm{s}^{5}\right.$ y $\left.2,4 \mathrm{ml} / \mathrm{s}^{7}\right)$ es escasa y la relevancia clínica de la mejora de la sintomatología $\left(3,6^{5}\right.$ y $2,8^{7}$ puntos) es moderada. Estos resultados coinciden con otros estudios de monoterapia con $\mathrm{AB}^{1-2}$. A nuestro entender, ningún tratamiento médico de la HBP consigue una mejoría objetiva del $\mathrm{Qmax}$, y en el caso de la sintomatología, la mejoría es superior con los $\mathrm{AB}$ que con los 5ARI. No obstante, la mejoría de síntomas objetivada en los diferentes ensayos clínicos parece inferior a la percepción subjetiva que se observa en la práctica clínica. Una explicación podría ser que las preguntas de los diferentes cuestionarios de puntuación de sintomatología (AUA o IPSS) no son capaces de reflejar la situación clínica real del paciente y por ello no captan de forma eficaz la mejora que experimentan los pacientes con el tratamiento.

En cuanto a la relevancia clínica de los resultados del estudio MTOPS ${ }^{9}$ se puede concluir que la progresión en HBP entendida como empeoramiento clínico o como aparición de complicaciones tiene una incidencia baja que va aumentando con el tiempo y que el tratamiento combinado consigue una reducción muy importante del riesgo de progresión con un número bajo de pacientes a tratar.

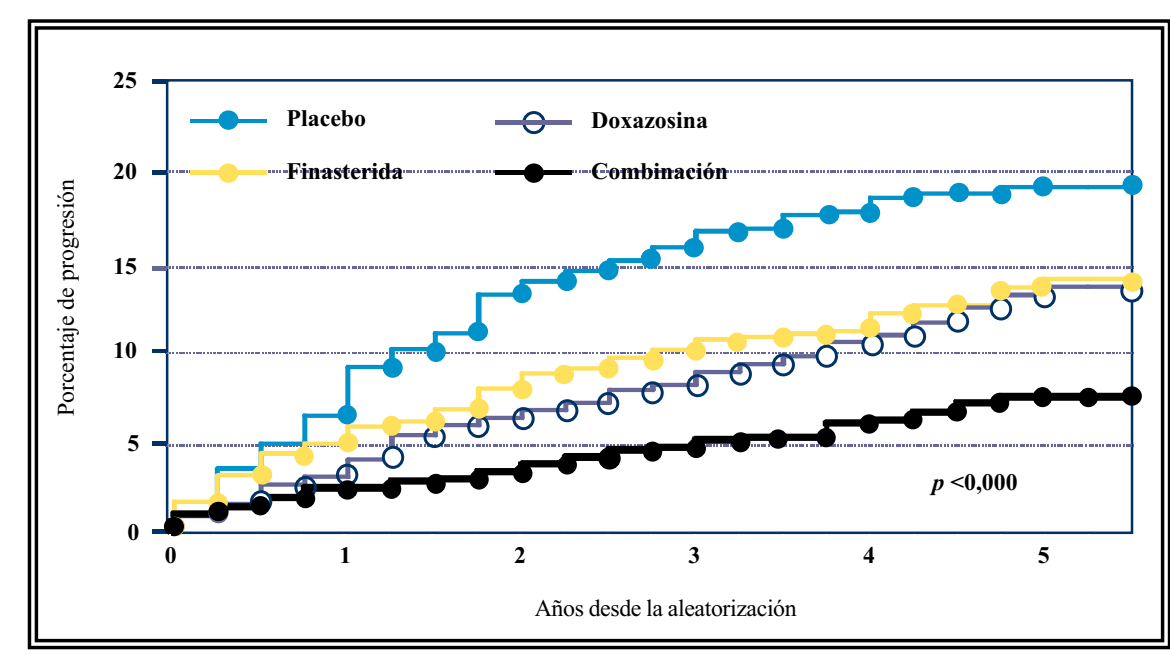

FIGURA 3. Porcentaje de progresión según grupos de tratamiento en el estudio MTOPS. 


\section{REFERENCIAS}

1. Lepor H. Phase III multicenter placebo-controlled study of tamsulosin in benign prostatic hyperplaisa. Urology 1998; 51(6): 892-900.

2. Roehrborn CG, Oesterling JE, Auerbach S y cols. The Hytrin community assessment trial study: a one-year study of terazosin versus placebo in the treatment of men with symptomatic benign prostatic hyperplasia. Urology 1996; 47(2): 159- 168.

3. McConnell JD, Bruskewitz R, Walsh P y cols. The effect of finasteride on the risk of acute urinary retention and the need for surgical treatment among men with benign prostatic hyperplasia. N Engl J Med 1998; 338(9): 557-563.

4. Roehrborn CG, Boyle PJ, Nickel C y cols. Efficacy and safety of a dual inhibitor of 5-alpha-reductase types 1 and 2 (dutasteride) in men with benign prostatic hyperplasia.

5. Lepor H, Williford WO, Barry MJ y cols. The efficacy of terazosin, finasteride, or both in benign prostatic hyperplasia. N Engl J Med 1996; 335(8): 533-539.

6. Debruyne FMJ, Jardin A, Colloi D y cols. Sustained-release alfuzosin, finasteride and the combination of both in the treatment of benign prostatic hyperplasia. Eur Urol 1998; 34: 169-175.

7. Kirby RS, Roehrborn C, Boyle P y cols. Efficacy and tolerability of doxazosin and finasteride, alone or in combination, in treatment of symptomatic benign prostatic hyperplasia: the Prospective European Doxazosin and Combination Therapy (PREDICT) trial. Kirby RS, Roehrborn C, Boyle P y cols. Urology 2003; 61(1): 119-126.

8. Barkin J, Guimar?es M, Jacobi G y cols. Alpha-blocker therapy can be withdrawn in the majority of men following initial combination therapy with the dual 5?-reductase inhibitor dutasteride. Eur Urol 2003; 44: 461-466.
9. Mc Connell JD, Roehrborn CG, Bautista OM y cols. The long-term effect of doxazosin, finasteride, and combination therapy on the clinical progression of benign prostatic hyperplasia. N Engl J Med 2003; 349(25): 2387-2398.

10. Sackett DL, Richardson WS, Rosenberg W, Haynes RB. Medicina Basada en la Evidencia. Cómo ejercer y enseñar la MBE. 1a ed. Madrid: Churchill Livingstone; 1997.

11. Millán Rodríguez F, Rodríguez Vallejo JM, Carballido Rodríguez J. Interpretación de resultados de ensayos clínicos en hiperplasia benigna prostática. Actas Urol Esp 2002; 26(1): 4-14.

12. Small Dr, Lanigan DJ, Khab AB y cols. Comparison of patients' assessment of urinary flow strength with uroflowmetry. Eur Urol 1997; 31(2): 148-152.

13. Cummings SR, Black DM, Thompson DE y cols. Effect of alendronate on risk of fracture in women with low bone density but without vertebral fractures: results from the Fracture Intervention Trial. JAMA 1998; 280(24): 20772082.

14. Pedersen TR, Kjekshus J, Berg K y cols. Randomised trial of colesterol lowering in 4444 patients with coronary Herat disease: the Scandinavian Simvastatin Survival Study (4S). Lancet 1994; 344(8934):1383-1389.

\author{
Dr. F. Millán Rodríguez \\ Servicio de Urología. Fundación Puigvert \\ Cartagena 340-350 \\ 08025 Barcelona \\ e-mail: fmillan@menta.net
}

(Trabajo recibido el 24 de junio 2005) 